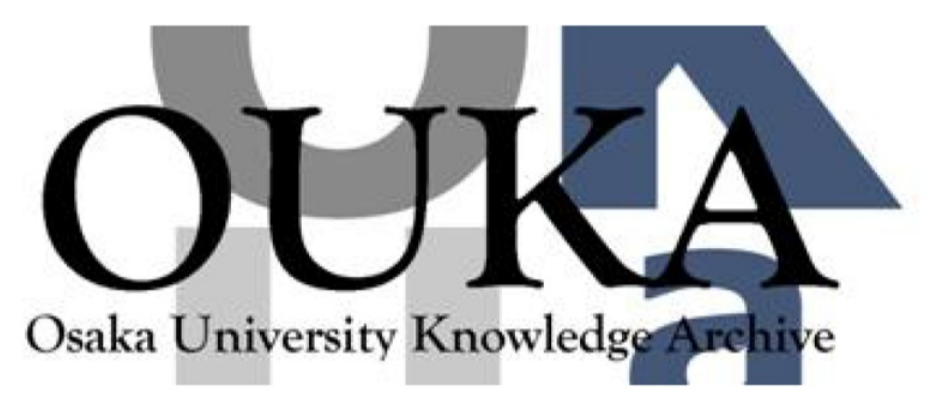

\begin{tabular}{|c|l|}
\hline Title & $\begin{array}{l}\text { Heat transfer and particle behaviours in } \\
\text { dispersed two-phase flow with different heat } \\
\text { conductivities for Liquid and solid }\end{array}$ \\
\hline Author(s) & $\begin{array}{l}\text { Tsutsumi, Takaaki; Takeuchi, Shintaro; } \\
\text { Kajishima, Takeo }\end{array}$ \\
\hline Citation & $\begin{array}{l}\text { Flow, Turbulence and Combustion. 92 }(1,2) \\
\text { p. 103-p. } 119\end{array}$ \\
\hline Issue Date & $2014-01$ \\
\hline oaire:version & AM \\
\hline URL & https://hdl. handle. net/11094/57202 \\
\hline rights & \\
\hline Note & \\
\hline
\end{tabular}

Osaka University Knowledge Archive : OUKA

https://ir. Library. osaka-u. ac. jp/

Osaka University 


\title{
Heat Transfer and Particle Behaviours in Dispersed Two-Phase Flow with Different Heat Conductivities for Liquid and Solid
}

\author{
Takaaki Tsutsumi ${ }^{1}$, Shintaro Takeuchi ${ }^{1}$ and Takeo Kajishima ${ }^{1}$ \\ ${ }^{1}$ Department of Mechanical Engineering, Osaka University \\ 2-1 Yamada-oka, Suita-city, Osaka 565-0871 Japan
}

\begin{abstract}
Liquid-solid two-phase flow with heat transfer is directly simulated, to investigate the effects of the ratios of heat conductivities (solid to liquid) and bulk solid volume fraction from dense to dilute situations. The interaction between fluid and particles is solved by our original immersed solid approach on a rectangular grid system. A discrete element method with a soft-sphere collision model is applied for particle-particle and particle-wall interactions. Governing equation of temperature is time-updated with an implicit treatment for the diffusion term, which enables robust simulation with particles of very high/low ratios of heat conductivities (from 1/1000 to 1000) to the fluid. The local heat flux at the fluid-solid interface is modelled by a new flux decomposition technique, and incorporated into the implicit scheme of the temperature. The method is applied to a 2-D particulate flow in a natural convection in a square domain at a relatively low Rayleigh number. In the dense condition, for the cases with high ratios of heat conductivity, the heat transfer is promoted by strong convection, while the particles of low ratios of heat conductivity tend to hinder the development of the temperature rise in the flow field, causing a weak convection and low Nusselt number. Under a condition of relatively low solid volume fraction, fixed particles only depress the heat convection as the number of particles and heat conductivity ratio increase. For the cases with freely-moving particles, on the other hand, heat conductivity of particles has a stronger influence on the heat transfer of the system than the number of particles. The above simulation results highlight the effect of temperature distributions within the particles and liquid.
\end{abstract}

keywords: Multiphase flow, Solid dispersion, Immersed solid object, Conjugate heat transfer, Heat conductivity ratio 


\section{Introduction}

Solid-dispersed two phase flows often involve heat (and mass) transfer through the interface. Temperature distributions in both fluid and solid phases play important roles on the fluid-solid interaction.

In early studies of theoretical and numerical modelling of particulate flows, especially for large systems, heat and mass transfer problems in dispersed two-phase flows are often treated as a homogeneous media or continuous and fully inter-penetrating two-fluid media [1]. In such cases with a large number of particles, most of the existing approaches assume a unique temperature for each point-source particle and simplify the heat transfer process at the fluid-solid interface.

However, local temperature distribution and flux between particle and fluid are known to affect many reaction processes. Examples include the fluidazation of functional heating medium to be applied for pharmaceutical process and heat storage system. Also in the fields of chemical, mineral and petroleum engineerings, the heat transfer between catalyst particle and reactive product is vital for the stable chemical reaction. To understand the heat transfer mechanism in fluid-solid two-phase flow, it is important to consider the fluid-particle interaction and temperature distributions within both solid and fluid phases, especially for finite-sized solid objects.

Numerical study of heat transfer problem in a flow including solid objects has been attempted by many researchers. Pioneering work was done by MaKenna et al. [2]. They employed unstructured fluid mesh that fits the surface of the solid object, and studied the size effect of catalyst particles (fixed in the domain) on the heat exchange with the surrounding fluid. Gan and co-workers [3; 4] reported 2-D flow with particles settling and melting in a hot fluid of the same material and showed characteristic lateral oscillations of the settling isothermal particles. Yu et al. [5] simulated heat transfer between fluid and freely-moving particles by using their distributed Lagrangian multiplier method. They applied the method to analyse the heat transfer in a uniform flow including nanofluids and sheared non-colloidal suspensions. Their study shows that the difference in temperature distribution within the particle changes the heat transfer performance of the system. More fundamental computational studies focus on the boundary conditions for the heat transfer on the particle surface with immersed boundary techniques. Iaccarino and Moreau [6] proposed a secondorder interpolation scheme (linear in the tangential direction and quadratic in the normal direction) for immersed boundary problems. Kim et al. [7] and Kim and Choi [8] proposed a heat source/sink method for imposing iso-thermal and iso-heat-flux boundary conditions at the immersed boundary of a fixed particle. Similar approaches were proposed and fully validated by Pacheco and co-workers [9; 10] and Ren et al. [11] with a fixed particle, and Zhang et al. [12] applied their heat source/sink approach to a heat transfer problem around an externally-oscillating cylinder. Ueyama et al. [13] developed a numerical method for fluidparticle interaction problem considering temperature distribution within the particles, and studied the effect 
of bulk solid volume fraction and particle arrangement (fixed array or freely-moving particles) on the heat transfer performance of the system.

Certainly, there are few previous studies that deal with a conjugate heat transfer problem (convective and conductive heat transfers) in a two-phase flow including freely-moving particles.

In the present work, we study a heat transfer problem in a multiphase flow of dispersed solid particles including the effect of local heat flux at the liquid-solid surface (and therefore temperature gradient within the solid object). To facilitate the treatment of interaction problem between fluid and a large number of relatively moving particles, a fixed grid approach is adopted. The interaction between fluid and particles is solved with our original immersed solid approach $[14 ; 15]$ on a rectangular grid system. The method employs a simple procedure for the momentum exchange by imposing a volume force (as an interaction force) on both solid and fluid phases. The method has been applied for studying a clustering process with a total of 1000 spherical particles in a turbulent flow [14;16;17]. Also the usefulness of our method has been demonstrated by Nishiura et al. [18] through the analysis of sedimentation process employing a total of $10^{5}$ spherical particles.

To include the effect of conjugate heat transfer in a liquid-solid interaction problem, a heat flux decomposition model is proposed for the heat conduction at the liquid-solid interface. The model employs a procedure for solving the temperature field in an Eulerian coordinate system by considering the interface direction, and it shows good compatibility with our immersed solid approach. The contact of particles may become one of the important paths for heat transfer in particulate flows, and heat exchange between the particles is restricted by heat resistance at the surface contacting point. This problem is still explored from micro and macroscopic views. One of the existing approaches considers the effect of heat resistance with surface roughness by attaching small grains on the surface of the particle [19]. However, in the present work, to simplify the phenomenon, only the repulsion is modelled by a discrete element method [20] with a soft-sphere collision model, and the effect of the heat fluxes between touching particles and particle-wall is excluded to focus on the heat transfer purely via fluid convection and conduction within each particle.

The present method is applied to a direct numerical simulation of 2-D laminar natural convection of relatively low Rayleigh number in a confined square domain including multiple particles of round shape. By including the particles of different ratios of heat conductivities (solid to liquid), we look into the effect of the solid temperature distribution on the behaviour of the particles, and the heat transfer mechanism is studied in the solid-dispersed two-phase flow for different bulk solid volume fractions. 


\section{Governing Equations and Numerical Methods}

\subsection{Governing equations}

The governing equations for fluid are the equations of continuity, momentum and energy:

$$
\begin{aligned}
& \nabla \cdot \boldsymbol{u}_{f}=0, \\
& \rho_{f} \frac{\partial \boldsymbol{u}_{f}}{\partial t}+\rho_{f} \boldsymbol{u}_{f} \cdot \nabla \boldsymbol{u}_{f}=-\nabla p+\mu_{f} \nabla^{2} \boldsymbol{u}_{f}+\rho_{f} \beta\left(T-T_{0}\right) \boldsymbol{g}, \\
& \frac{\partial \rho_{f} c_{f} T_{f}}{\partial t}+\boldsymbol{u}_{f} \cdot \nabla\left(\rho_{f} c_{f} T_{f}\right)=\nabla \cdot\left(\lambda_{f} \nabla T_{f}\right),
\end{aligned}
$$

where $\boldsymbol{u}_{f}$ is the fluid velocity, $\rho_{f}$ the reference density, $\boldsymbol{g}$ the gravitational acceleration, $\beta$ the coefficient of thermal expansion, $T_{f}$ the fluid temperature, $T_{0}$ the reference temperature, $c_{f}$ the specific heat. Here, an incompressible fluid is assumed and Boussinesq approximation is employed to include the effect of density fluctuation. In the following, viscous coefficient $\left(\mu_{f}\right)$, heat capacity per unit volume $\left(\rho_{f} c_{f}\right)$ and thermal conductivity $\left(\lambda_{f}\right)$ are assumed to be constant.

Governing equations for motion of the solid object are Newton's equations for momentum and angular momentum:

$$
m_{p} \frac{d \boldsymbol{v}_{p}}{d t}=\boldsymbol{F}+\boldsymbol{G}_{p}, \quad \boldsymbol{I}_{p} \frac{d \boldsymbol{\omega}_{p}}{d t}=\boldsymbol{T}+\boldsymbol{N}_{p}
$$

where $m_{p}$ is the mass, $\boldsymbol{I}_{p}$ the inertia tensor, $\boldsymbol{v}_{p}$ the translating velocity, $\boldsymbol{\omega}_{p}$ the angular velocity, $\boldsymbol{F}$ and $\boldsymbol{T}$ the hydrodynamic force and its moment, respectively, and $\boldsymbol{G}_{p}$ and $\boldsymbol{N}_{p}$ are the external force and torque, respectively.

\subsection{Fluid-solid interaction model: immersed solid approach}

Momentum exchange at the fluid-solid interface, where a cell is partially occupied by a solid object, is solved by the immersed solid approach developed by Kajishima and the co-workers [14; 16; 17], on a uniformly distributed fixed grid system. This is briefly described below.

A velocity field $\boldsymbol{u}$ is established through volume-averaging of the local fluid velocity $\boldsymbol{u}_{f}$ and the local particle velocity $\boldsymbol{u}_{p}$ in a cell:

$$
\boldsymbol{u}=(1-\alpha) \boldsymbol{u}_{f}+\alpha \boldsymbol{u}_{p}
$$

where $\alpha(0 \leq \alpha \leq 1)$ is the local solid volume fraction in the cell. The particle velocity $\boldsymbol{u}_{p}$ is decomposed into translating and rotating components as $\boldsymbol{u}_{p}=\boldsymbol{v}_{p}+\boldsymbol{\omega}_{p} \times \boldsymbol{r}$. This mixture velocity field $\boldsymbol{u}$ is assumed to obey the following equation:

$$
\frac{\partial \boldsymbol{u}}{\partial t}=-\nabla p / \rho_{f}+\boldsymbol{H}_{u}+\beta\left(T-T_{0}\right) \boldsymbol{g}+\boldsymbol{f}_{p}
$$


where $\boldsymbol{H}_{u}$ contains the convective and viscous terms. Interaction term $\boldsymbol{f}_{p}$ works to assign the mixture velocity that satisfies the non-slip boundary condition at the interface $[14 ; 16 ; 17]$. For spatial discretisation of Eq. (2), second-order finite difference schemes are used. For time-updating Eq. (2), the 2nd-order AdamsBashforth and Crank-Nicolson methods are employed for the convective and viscous terms, respectively. The pressure gradient term in Eq. (2) is treated implicitly by a fractional step method. With the corrected velocity field $\widetilde{\boldsymbol{u}}$, the fluid-solid interaction term $\boldsymbol{f}_{p}$ is modelled as follows:

$$
\boldsymbol{f}_{p}=\alpha \frac{\left(\boldsymbol{u}_{p}-\widetilde{\boldsymbol{u}}\right)}{\Delta t}
$$

where $\Delta t$ is the time increment.

For motion of the particles, the same force as Eq. (3) applies to the fraction of the solid in the cell with the opposite sign. The surface integration of the hydrodynamic forces is changed to the integration of $\boldsymbol{f}_{p}$ over the volume of the particle $V_{p}$ :

$$
\begin{aligned}
m_{p} \frac{\boldsymbol{v}_{p}^{n+1}-\boldsymbol{v}_{p}^{n}}{\Delta t} & =\int_{V_{p}}\left(-\rho_{f} \boldsymbol{f}_{p}\right) d V+\boldsymbol{G}_{p}, \\
\boldsymbol{I}_{p} \frac{\boldsymbol{\omega}_{p}^{n+1}-\boldsymbol{\omega}_{p}^{n}}{\Delta t} & =\int_{V_{p}} \boldsymbol{r} \times\left(-\rho_{f} \boldsymbol{f}_{p}\right) d V+\boldsymbol{N}_{p},
\end{aligned}
$$

where the superscripts represent time levels.

The above replacement from surface to volume integrations considerably facilitates the computation of the solid motion, and also the use of the same body force $f_{p}$ for both (fluid and solid) phases in a shared Cartesian cell ensures no leakage of momentum between the phases. Eqs. (4) and (5) are solved with a predictor-corrector method [13]. The new position of the particle is obtained after time-updating Eqs. (4) and (5), and the local solid volume fraction $\alpha$ and surface normal vector $\boldsymbol{n}$ (directing from particle to fluid) are obtained accordingly.

\subsection{Temperature field and interfacial heat conduction}

Temperature field is treated in an Eulerian way. The numerical simulation are conducted under $\rho_{s}=\rho_{f}(=$ $\rho)$ and $c_{s}=c_{f}(=c)$. The following equation is solved with the distributed material property $\lambda(\boldsymbol{x})$ :

$$
\begin{aligned}
\frac{\partial \rho c T}{\partial t}+\boldsymbol{u} \cdot \nabla(\rho c T) & =\nabla \cdot(-\boldsymbol{q}) \\
& =\nabla \cdot(\lambda \nabla T) .
\end{aligned}
$$

In the following, discretised cell temperature is represented by $T_{i j}=\left\langle X_{s} T_{s}+X_{f} T_{f}\right\rangle_{i j}$, where $X_{k}(k=$ $s, f)$ is the indicator function of $k$-phase, $\langle\cdot\rangle$ means the cell average and the subscripts $i j$ denote the twodimensional indices of the computational cell. The discretised temperature gradient $\left.\nabla T\right|_{i j}$ is decomposed 
into surface-normal component $\left.(\boldsymbol{n n}) \cdot \nabla T\right|_{i j}$ and tangential component $\left.(\boldsymbol{I}-\boldsymbol{n n}) \cdot \nabla T\right|_{i j}$, where $\boldsymbol{I}$ is the identity tensor and $\boldsymbol{n}$ is the unit outward normal vector at the object surface. In an interfacial cell partially occupied by the solid object (local solid volume fraction $\alpha$ ), the following mean heat conductivities are defined in the surface normal and tangential directions, respectively:

$$
\frac{1}{\lambda_{h}}=\frac{1-\alpha}{\lambda_{f}}+\frac{\alpha}{\lambda_{s}}, \quad \lambda_{a}=(1-\alpha) \lambda_{f}+\alpha \lambda_{s}
$$

Assuming that the solid and fluid temperatures match with each other at the interface, the heat flux at the interfacial cell $\boldsymbol{q}_{\text {surf }}$ is given by the following formula:

$$
\begin{aligned}
\boldsymbol{q}_{\mathrm{surf}} & =-\left.\lambda_{h}(\boldsymbol{n n}) \cdot \nabla T\right|_{i j}-\left.\lambda_{a}(\boldsymbol{I}-\boldsymbol{n n}) \cdot \nabla T\right|_{i j} \\
& =-\left[\lambda_{a} \boldsymbol{I}+\left(\lambda_{h}-\lambda_{a}\right)(\boldsymbol{n n})\right]_{i j} \cdot\left(\begin{array}{c}
\left(-T_{i-1 / 2, j}+T_{i+1 / 2, j}\right) / \Delta \\
\left(-T_{i, j-1 / 2}+T_{i, j+1 / 2}\right) / \Delta
\end{array}\right),
\end{aligned}
$$

where $\Delta$ is the uniform grid spacing. At the cell totally occupied by the fluid or solid object, the heat conductivity $\lambda$ is set to that of the material. On the other hand, at the fluid-solid interface, where a cell is partially occupied by a solid object, we apply Eq. (7) as the interfacial heat flux at the cell.

This treatment of the heat flux would be essential when the heat conductivities of fluid and solid are different by orders of magnitude, where the local tangential heat flux could be relatively large compared with the surface normal flux. Note that this treatment of heat flux at the surface shows good compatibility with our immersed solid approach; since the local solid volume fraction $\alpha$ distributes in the interfacial cells where the interaction term Eq. (3) is calculated, then the heat flux is simply constructed by Eq. (7) with $\boldsymbol{n}$. In the following sections, the above formula is applied to circular geometry. However, the formula is also applicable to solid objects of arbitrary geometry with an algorithm of calculating $\alpha$ for non-circular/nonspherical objects [15].

Eq. (6) is time-updated with the Crank-Nicolson method for the diffusion term with the treatment of Eq. (7). This semi-implicit scheme stabilises the computation and enables simulation with particles of very high/low heat conductivities. 


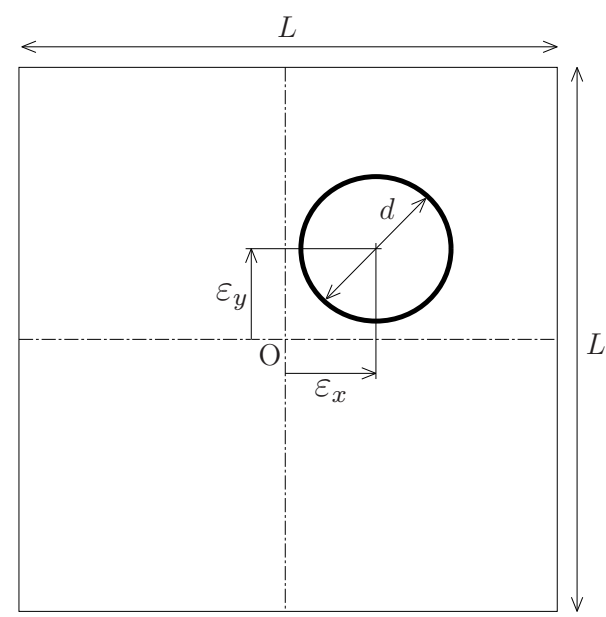

Figure 1: Schematic of off-centre cylinder in a square domain.

\subsection{Interparticle and particle-wall collision model}

A soft-sphere model is used to allow multiple-body collisions for interparticle and particle-wall collisions. A spring and dashpot model is employed to calculate the contact forces. In the model, the spring constant determines the characteristic time scale. The larger the spring constant is, the smaller the time step should be. In the present study, the same parameter values as Tsuji et al. [20] are used for the spring constant, restitution coefficient and friction coefficient.

Tsuji et al. [20] suggested the following condition for determining the time increment to sufficiently resolve the eigen oscillation of the mass-spring system:

$$
\Delta t \leq \frac{\pi}{n} \sqrt{\frac{m_{p}}{k}} \quad(n \geq 10) .
$$

In the present study, the smallest value of the right hand side of the above equation is found to be $1.47 \times 10^{-3}$ for the smallest $m_{p}$ employed in the following section. Therefore, considering the numerical accuracy of the momentum and energy equations from our preliminary study, the time increment is fixed to $\Delta t=5.0 \times 10^{-4}$, hereafter. In the present study, the largest CFL number based on this time increment is found to be $1.01 \times 10^{-2}$, and the CFL condition is less influential in comparison with the condition of Eq. (8).

In the present study, no heat exchange or heat source is modelled in the interparticle collisions, and interparticle cohesive force is assumed to be negligible. 


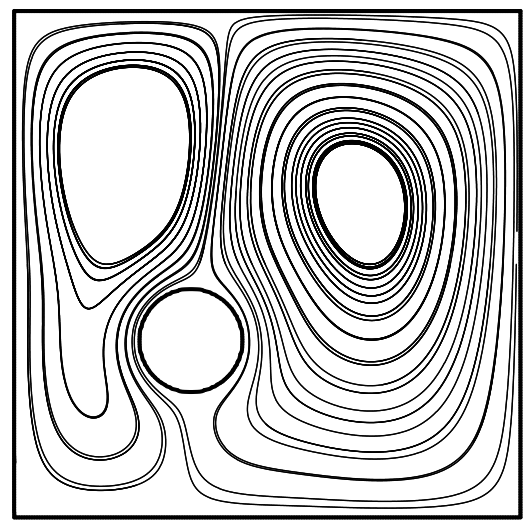

(a) Streamlines, $\mathrm{Ra}=10^{5}$

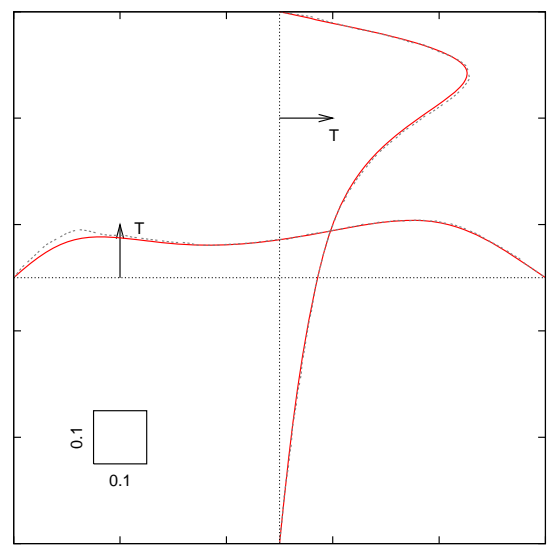

(c) Temperature, $\mathrm{Ra}=10^{5}$

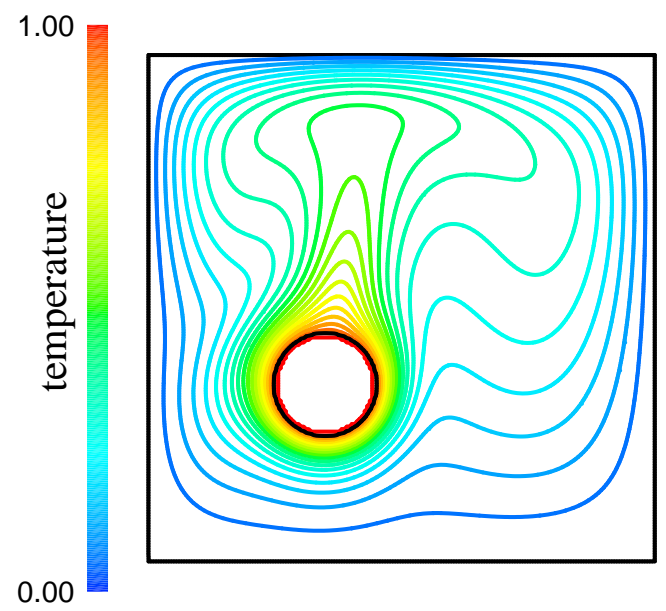

(b) Iso-thermal lines, $\mathrm{Ra}=10^{5}$

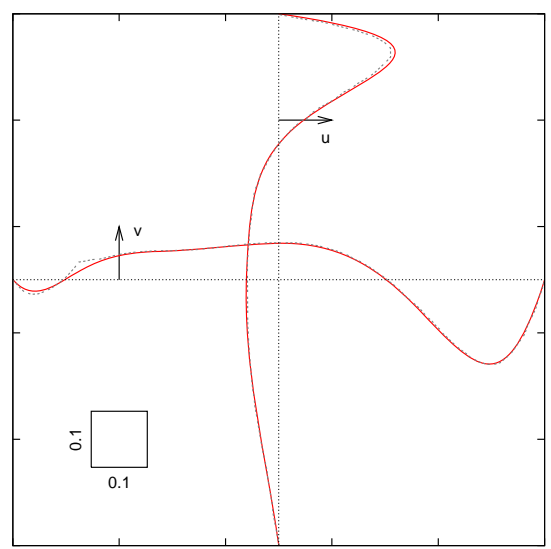

(d) Velocity, $\mathrm{Ra}=10^{5}$

Figure 2: (a)(b)Streamlines and iso-contours of temperature in a square region with an off-centre cylinder of $d=0.2 L$ placed at $\varepsilon_{x}=-0.15 L, \varepsilon_{y}=-0.15 L$. (c)(d)Temperature and velocity profiles on the vertical and horizontal centrelines for a natural convection in a square domain with a heated cylinder of $d=0.2 L$ placed at $\varepsilon_{x}=-0.3 L$ and $\varepsilon_{y}=0.3 L$. The solid lines show the present simulation result and the dotted lines are the result by Moukalled and Darwish [21]. Velocities are normalised by $\sqrt{g \beta \Delta T L}$.

\subsection{Verification: natural convection with a fixed particle}

The proposed method is applied to a problem of laminar natural convection from a heated cylinder placed off-centre of a square domain (side length $L$ ) as illustrated in Figure 1. The cylinder has a diameter $d=0.2 L$, and its centre is located at $\left(\varepsilon_{x}, \varepsilon_{y}\right)$ with respect to the domain centre. The cylinder has a nondimensional wall temperature $T=1$, and the surrounding four walls are maintained at $T=0$. Rayleigh number is $10^{5}$. The number of grid point is 200 in both horizontal and vertical directions.

Figures 2(a) and (b) show streamlines and isotherms. Two well-defined rotating vortices are observed from the streamlines. From the temperature contours, a strong thermal plume rises slightly in the left direction. The same natural convection problem has been studied by Moukalled and Darwish [21] using a 


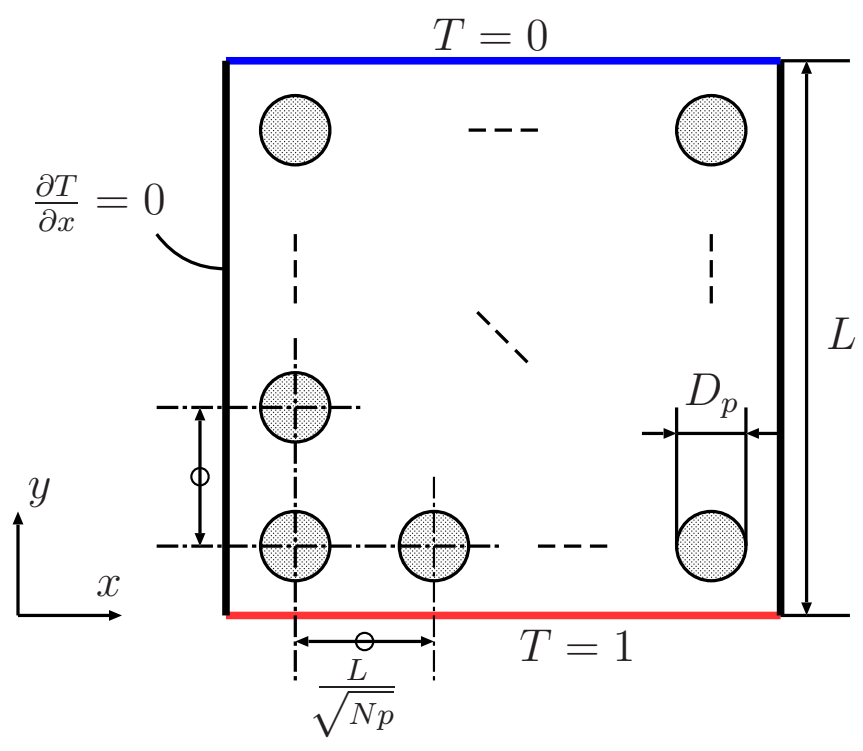

Figure 3: Schematic of the initial arrangement of particles.

bounded skew central-difference scheme, Sadat and Couturier [22] with a meshless diffuse approximation method, Pan [23] with an unstructured-Cartesian-mesh immersed boundary method and Pacheco-Vega et al. [10] using a direct forcing fixed-grid method. From the comparison, the present numerical results agree well with those presented in the above literatures.

Figures 2(c) and (d) show the temperature and velocity distributions on the vertical and horizontal centrelines for the same Rayleigh number. The result is compared with those by Moukalled and Darwish [21], and the temperature and velocities show reasonable agreement with each other. Together with the validation of Eq. (7) reported in [24], the applicability of the present method is established, and the present method is applied to a thermal flow problem developing around a particle in the following section.

\section{Results and Discussion}

Solid-dispersed two-phase flow under natural convection is studied for different heat conductivity ratios (solid to fluid) and solid volume fractions. The non-dimensional numbers used are Rayleigh number (Ra) and Prandtl number (Pr).

In the present work, the computational domain is a square shape of side length $L$, and the particles are initially arranged regularly in the domain as illustrated in Figure 3. Temperature difference between the top and bottom walls is kept constant $(\Delta T=1)$, and no heat flux is given at the lateral walls. The no-slip boundary condition and the Neumann condition are applied for the velocity and pressure, respectively, on the solid walls. The equations are non-dimensionalised with the reference length $L$, the reference velocity $U=\sqrt{g \beta \Delta T L}$, the reference pressure $\rho_{f} U^{2}$ and the characteristic temperature difference $\Delta T$. The ratio 


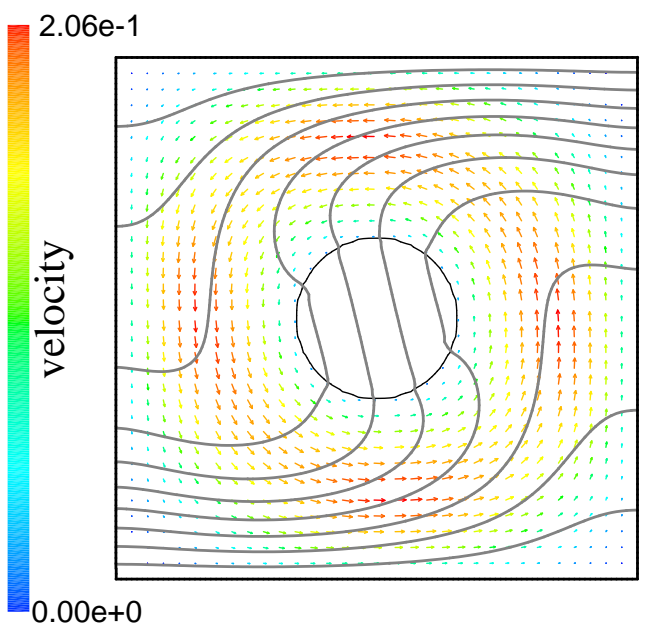

(a) $\lambda_{s} / \lambda_{f}=10^{-2}$

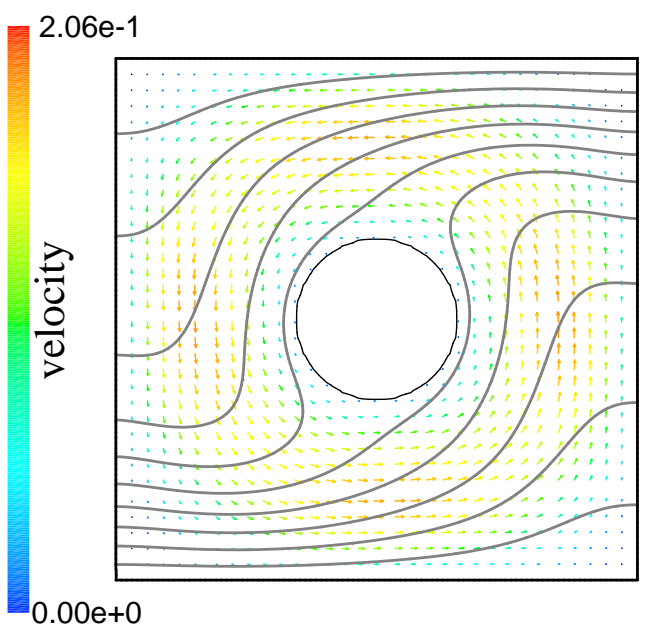

(b) $\lambda_{s} / \lambda_{f}=10^{2}$

Figure 4: Instantaneous flow field $(t=1500)$ of Rayleigh number $10^{4}$ including a single particle of (a) $\lambda_{s} / \lambda_{f}=10^{-2}$ and (b) $\lambda_{s} / \lambda_{f}=10^{2}$. Colour shows the magnitude of the fluid velocity and iso-contours of temperature are plotted at constant intervals. The particle is fixed at the domain centre and the surface is represented with a black line.

Table 1: Simulation parameters (dense case).

\begin{tabular}{ccc}
\hline Num. of grid points & $N_{x} \times N_{y}$ & $200 \times 200$ \\
Spatial resolution & $L / \Delta$ & 200 \\
Number of particles & $N_{p}$ & $14^{2}$ \\
Diameter of particles & $D_{p}$ & $0.05 L$ \\
Rayleigh number & Ra & $1 \times 10^{5}$ \\
Heat conductivity ratio & $\lambda_{s} / \lambda_{f}$ & $10^{-3}, 10^{0}, 10^{1}, 10^{2}, 10^{3}$ \\
\hline
\end{tabular}

of the particle diameter to grid spacing is $D_{p} / \Delta=10$, which allows reasonable accuracy for the drag coefficient in the present particle Reynolds number range [14;25]. And the grid dependency for the heat conduction model is verified in [24]. To investigate the effect of natural convection on the particle behaviour and heat transfer, Prandtl number, density ratio and specific heat ratio are set to unity.

Prior to the study of behaviour of freely-moving particles, Figure 4 shows two typical snapshots of the flow fields around a particle fixed at the centre of the domain under the above boundary conditions. For the case employing particles of a low conductivity ratio $\left(\lambda_{s} / \lambda_{f}=10^{-2}\right)$ in Figure 4(a), once a large temperature gradient develops within the particle, it takes a long time until the temperature imbalance is resolved. On the other hand, a nearly flat temperature distribution immediately develops within the particle of a high conductivity $\left(\lambda_{s} / \lambda_{f}=10^{2}\right)$ as shown in Figure 4(b). Similar temperature and velocity distributions are reported by Yu et al. [5] in a shear flow with a fixed particle.

In the following, interaction between the fluid and particles is simulated with different ratios of heat conductivity and solid volume fractions. 


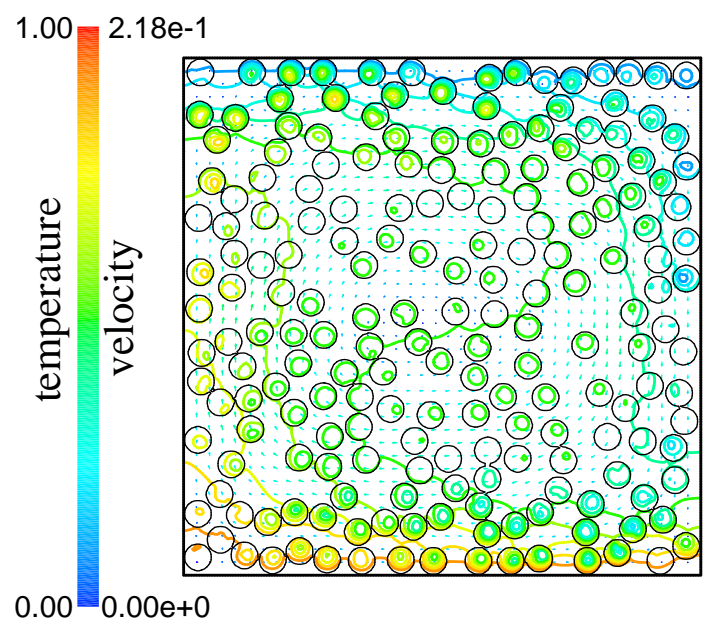

(a) $\lambda_{s} / \lambda_{f}=10^{-3}$

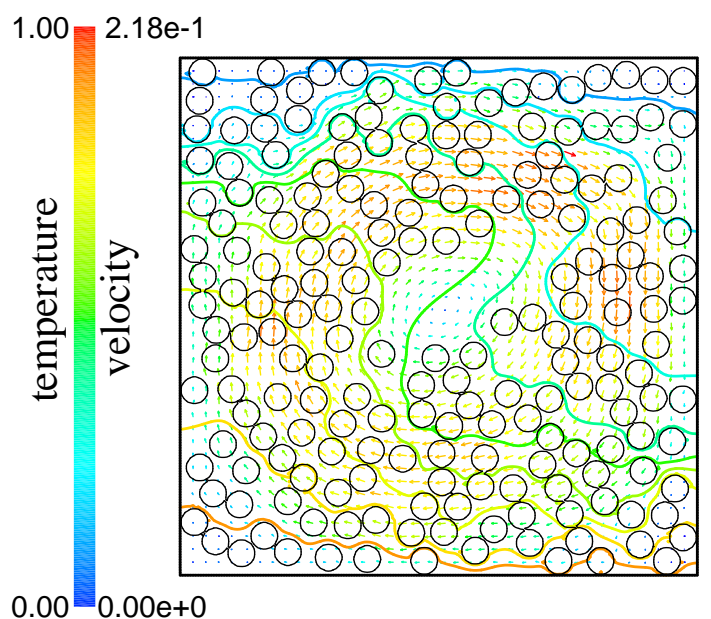

(b) $\lambda_{s} / \lambda_{f}=10^{3}$

Figure 5: Instantaneous flow field and contours of temperature for different heat conductivities. Rayleigh number is $10^{5}$ and bulk solid volume fraction is $38.5 \%$. Colour shows the magnitude of the fluid velocity and iso-contour of temperature.

\subsection{Effect of heat conductivity ratio of solid to fluid}

We look into the effect of the heat conductivity ratio on the particle motion in a natural convection of Rayleigh number $10^{5}$. The corresponding particle Reynolds number (based on $D_{p}$ and the natural convection speed driven by the vertical temperature difference across $D_{p}$ ) is approximately 80 at most for a fixed particle. The other parameters are summarised in Table 1. The bulk solid volume fraction is $38.5 \%$.

Figure 5 shows instantaneous flow and temperature fields at $t=1500$ by employing the particles of $\lambda_{s} / \lambda_{f}=10^{-3}$ and $10^{3}$. In both cases, the particles and fluid are found to constantly circulate in one direction around the domain centre after the initial developing stage. However, the local concentrations of the particles and time-averaged flow fields are different for the two cases. Figure 6 compares the time-averaged temperature and velocity fields for the two heat conductivity ratios.

For the particles of $\lambda_{s} / \lambda_{f}=10^{-3}$, the temperature gradient within the particle hardly re-distributes within the particle or to the fluid. The concentrated isothermal lines near the top and bottom walls in Figure 6(a) suggest that, once a layer of particles is formed in those regions, the heat transfer from/to the wall is blocked by the particles of poor-conductivity, resulting in poor development of the temperature gradient in the fluid phase (and in turn, weak buoyancy force). In addition, the particles of low heat conductivity take a long time to fall/rise after cooled/heated by the top/bottom wall. Therefore, low heat conduction in the particle could cause weak fluid convection.

On the other hand, for the case of $\lambda_{s} / \lambda_{f}=10^{3}$, the particles near the hot wall transfer the heat to the fluid and the particles near the cold wall efficiently release the heat to the top wall from the fluid. The generated temperature gradient in the fluid phase causes fluid convection as observed in Figure 6(b), and a circulating 


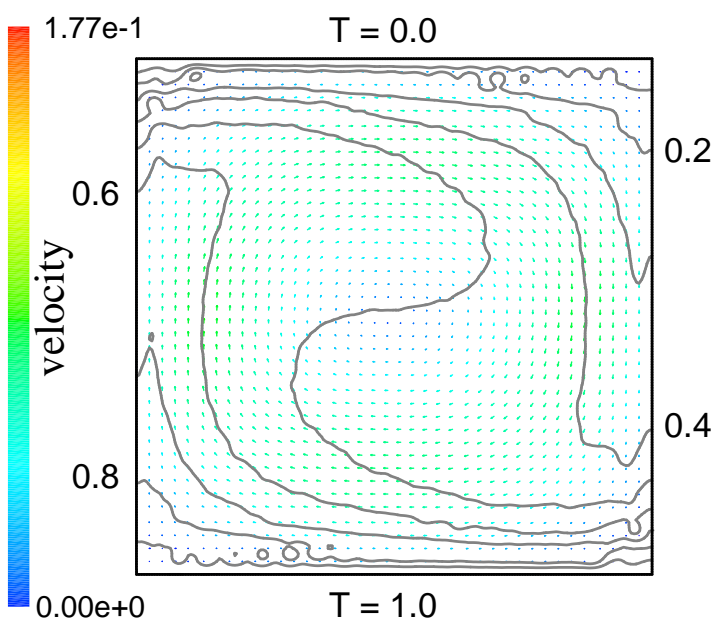

(a) $\lambda_{s} / \lambda_{f}=10^{-3}$

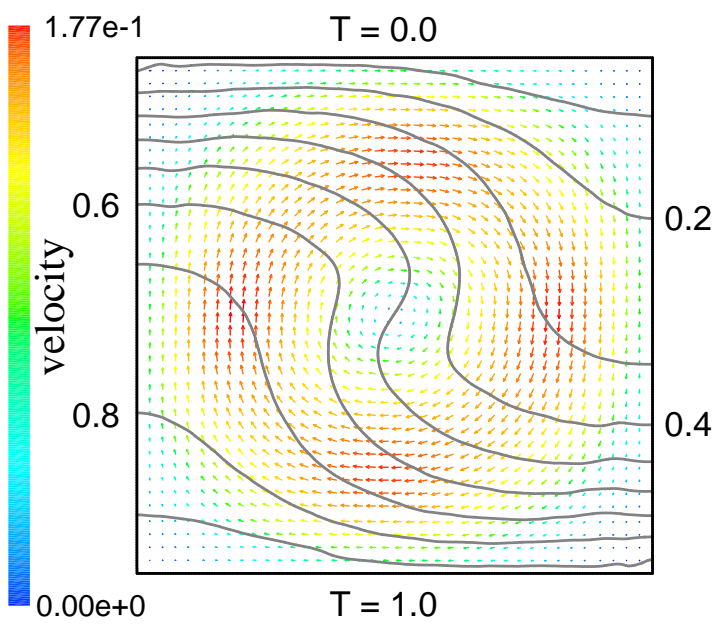

(b) $\lambda_{s} / \lambda_{f}=10^{3}$

Figure 6: Time-averaged temperature and velocity fields for different heat conductivities. Rayleigh number is $10^{5}$. Average is taken between $t=500$ and 1500. Magnitude the fluid velocity are levelled by colour, and iso-contours of temperature are plotted at constant intervals.

flow of the heated particles further promotes the heat transfer of the system. It is also characteristic, from Figure 5(b), that a region of low number density of the particles is found near the domain centre due to high rotating speed of the particulate flow.

The heat transfer rate in two-phase natural convection system is compared for different particle conductivities. For evaluating heat transfer rate, the following Nusselt number is used with the heat flux at the hot (bottom) wall:

$$
\mathrm{Nu}=\frac{1}{\Delta T} \int_{0}^{L}\left(-\frac{\partial T}{\partial y}\right)_{y=0} d x .
$$

Figure 7 compares the time evolutions of Nusselt number for three different $\lambda_{s} / \lambda_{f}$ cases. The case of the lowest conductivity ratio $\left(\lambda_{s} / \lambda_{f}=10^{-3}\right)$ exhibits very low Nusselt number over the time range tested, as expected. The profiles of $\lambda_{s} / \lambda_{f}=10^{1}$ and $10^{2}$ (not shown here) fall between the curves of "without particles" and $\lambda_{s} / \lambda_{f}=10^{3}$, and the average level of the Nusselt number is found to increase with increasing the heat conductivity ratio. The oscillatory behaviour of the Nusselt number is due to the fluctuation of the number density distribution of particles. The concentration of particles in the vicinity of conductive walls affects the instantaneous heat transfer. In case of employing high conductive particles, this tendency is enhanced resulting in the larger fluctuation of the Nusselt number.

The above results show that the solid heat conductivity largely influences the flow pattern in soliddispersed two-phase flows and that the circulation of the particles enhances the heat transfer rate by transporting the heat from bottom to top. 


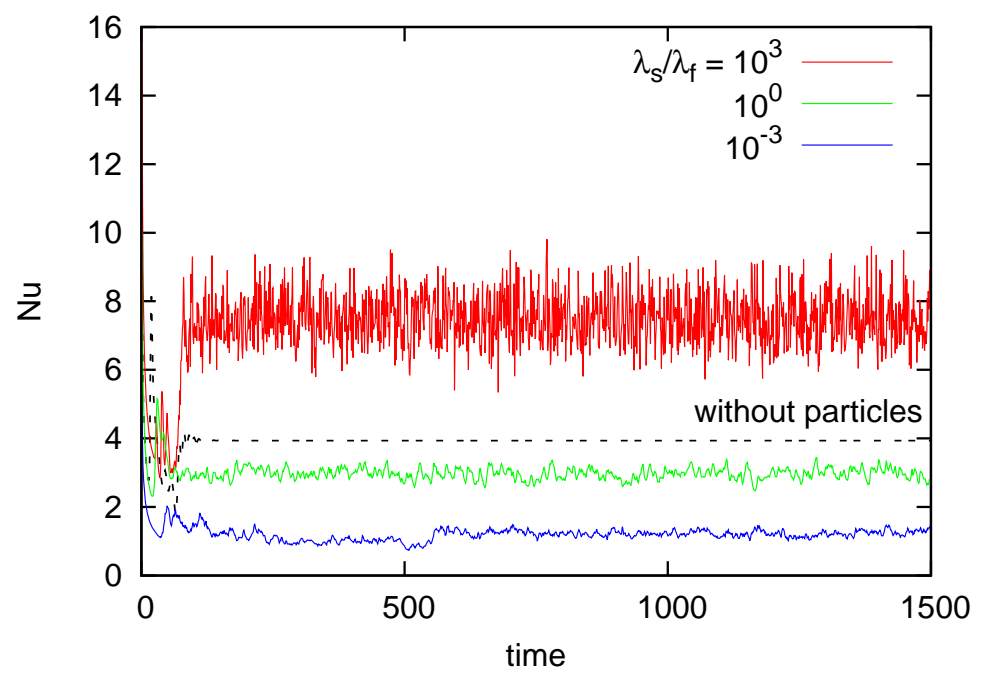

Figure 7: Comparison of time evolutions of Nusselt number for different ratios of heat conductivity. Solid volume fraction is $38.5 \%$

Table 2: Simulation parameters (dilute case).

\begin{tabular}{ccc}
\hline Num. of particles & $N_{p}$ & $0,1,2^{2}, 3^{2}, 5^{2}, 6^{2}, 10^{2}$ \\
Diameter of particles & $D_{p}$ & $\frac{0.3 L}{\sqrt{N p}}$ \\
Rayleigh number & Ra & $1 \times 10^{5}$ \\
Heat conductivity ratio & $\lambda_{s} / \lambda_{f}$ & $10^{-2}, 10^{0}, 10^{1}, 10^{2}$ \\
\hline
\end{tabular}

\subsection{Effect of the particle size}

Heat transfer performance of the system is compared for different particle sizes. The particle sizes are varied by keeping the bulk solid volume fraction $7.1 \%$. Therefore, the heat capacities of the individual particles for each case are different, but the bulk heat capacity of the solids is the same. To investigate the effect of the motion of the particles, two cases employing fixed particles and freely-moving particles are tested. The spatial resolution is fixed to $D_{p} / \Delta=10$ (except for the cases of $N_{p}=1$ and $2^{2}$, which are $D_{p} / \Delta=30$ and 20 , respectively), and the number of grid points along each side of the domain $(L / \Delta)$ is changed accordingly. The other simulation parameters are summarised in Table 2.

In the cases with fixed particles, steady flow and temperature fields develop. And, in all the cases with freely-moving particles, the fluid and particles are observed to steadily go around the domain centre.

Figure 8 shows the average Nusselt number, and Figure 9 shows the root mean square (r.m.s.) of the magnitude of velocity to see the development of convection of the fluid. Time-average is taken in the range from the time reached quasi-steady state to $t=1500$.

In Figure 8(a) (the cases with fixed particles), only the configuration of $N_{p}=2^{2}$ shows an improved heat transfer rate than the case without particles. After taking the maximum Nusselt numbers at $N_{p}=2^{2}$ for all the heat conductivity cases, an overall decreasing trend of the average Nusselt number is observed with increasing the number of particles. Interestingly, at $N_{p}=2^{2}$ and $3^{2}$, the average Nusselt numbers for 


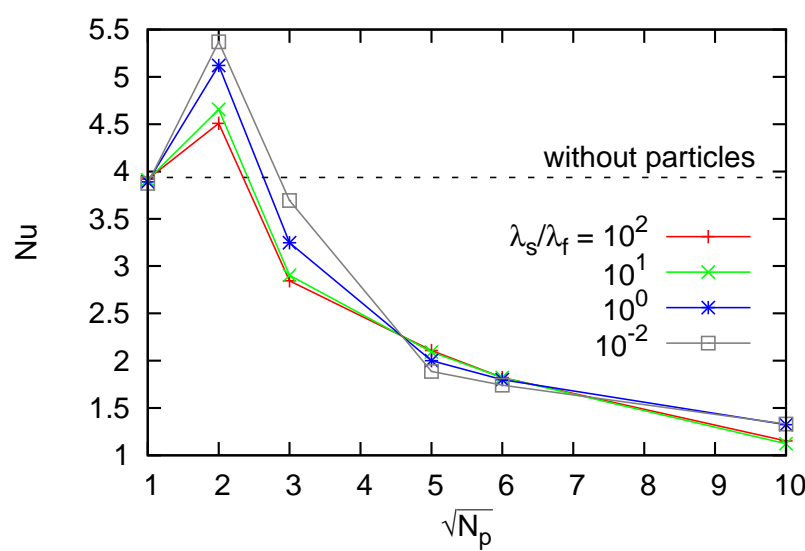

(a) Fixed particle cases

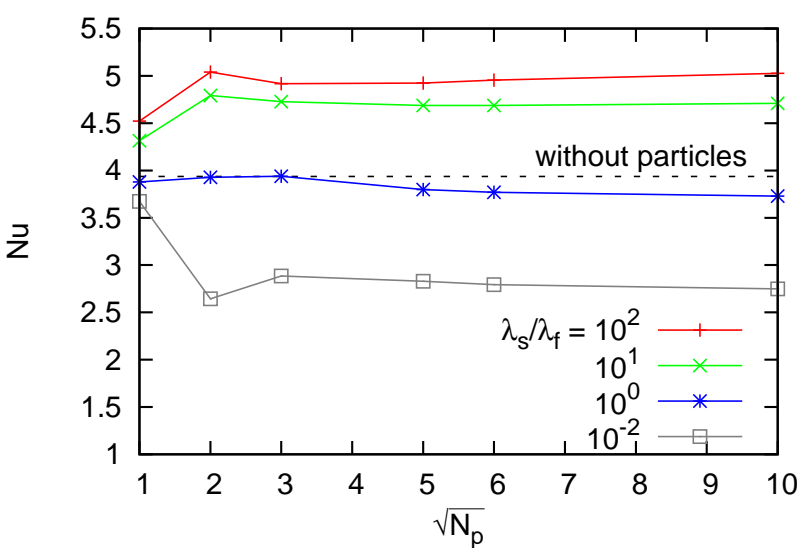

(b) Moving particle cases

Figure 8: Average Nusselt number as a function of number of particles for different heat conductivity ratios.

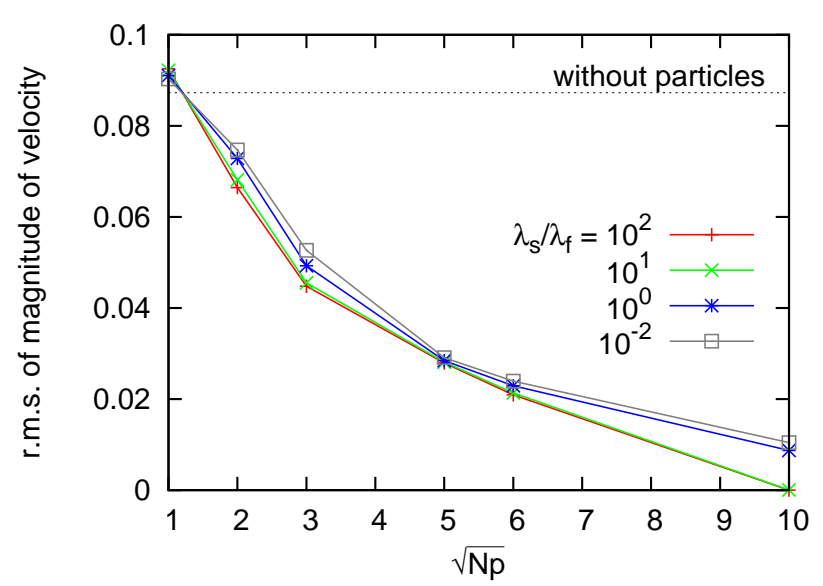

(a) Fixed particle cases

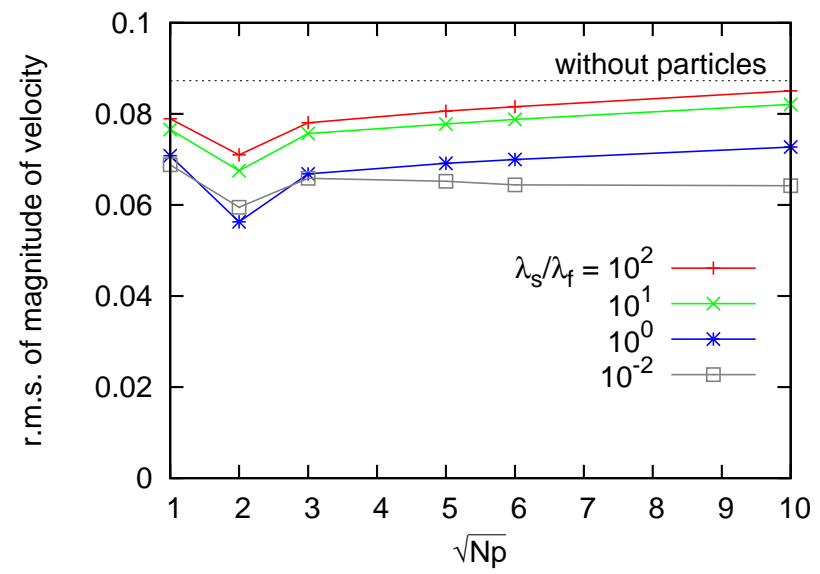

(b) Moving particle cases

Figure 9: Average root mean square of velocity as a function of number of particles for different heat conductivity ratios.

$\lambda_{s} / \lambda_{f}=10^{-2}$ and $10^{0}$ are larger than those of the higher heat conductivity ratios. This is explained that, due to the poor heat conduction in the fixed particles of the low heat conductivities, most of the energy input from the hot wall is transferred to the fluid for the low conductivity cases. The r.m.s. values at $N_{p}=2^{2}$ and $3^{2}$ in Figure 9(a) also show this. However, from Figure 9(a), the fixed array of particles works to hinder the development of the fluid convection as the number of particles and the heat conductivity ratio increase. These results suggest a possibility of promoting the heat transfer of the system by a proper choice of the number and arrangement of fixed particles.

In the cases with freely-moving particles, on the other hand, Figure 8(b) shows a higher Nusselt number for a case with a higher $\lambda_{s} / \lambda_{f}$. However, the Nusselt numbers are found to be insensitive to the number of particles (in the range $\sqrt{N_{p}}>3$ ) for each heat conductivity ratio. Especially for the case with particles of the same heat conductivity as the fluid $\left(\lambda_{s} / \lambda_{f}=10^{0}\right)$, the average Nusselt number is almost the same level as the case without particles. These results seem that, for the cases with freely-moving particles, heat 
conductivity of the particles has a stronger influence on the heat transfer of the system than the number of particles.

From Figures 8(a) and 9(a), for the cases with fixed particles, the heat convection is depressed and the heat transfer of the system decreases in proportion to the number of particles. However, from Figure 9(b), the levels of r.m.s. are also in accordance with the heat conductivity ratio, and only slight changes of the r.m.s. values are found with the number of particle (except for the case of $\sqrt{N_{p}}=2$ ). Figures 8(b) and 9(b) indicate that the convection is the major heat transfer means for the case of low $\lambda_{s} / \lambda_{f}$, and that, as $\lambda_{s} / \lambda_{f}$ is increased, the contributions of the convection (Figure 9(b)) and the heat conduction (Figure 8(b)) increase in the dilute conditions of the present study.

\section{Conclusions}

To simulate solid-dispersed two-phase flow with heat transfer, a method considering the effects of temperature distribution within a particle was developed based on a discrete-element and our original immersed solid methods. The proposed method is verified by solving the problem of natural convection in a square domain with a heated cylinder.

The method is applied to a 2-D liquid-solid two-phase flow from dilute to dense solid concentrations under a relatively low Rayleigh number. In the dense condition with freely-moving particles, the particles of a high heat conductivity induce the heat convection of the fluid, and promote the heat transfer of the system. On the other hand, for particles of a low heat conductivity, the particles move slowly and concentrate in the near-wall regions. Those particles tend to intercept the heat transfer from the hot wall to the fluid, resulting in low Nusselt number. In the dilute condition, fixed particles hinder the development of the heat convection for each heat conductivity ratio as the number of particles increases. For freely-moving particles, the Nusselt number and r.m.s. of the magnitude of the velocity are found to be less sensitive to the number of particles, whereas the heat conductivity ratio has a stronger influence on the heat transfer of the system. The results indicate that, in the dilute conditions, the convection is the major contributor for the heat transfer in the system of both fixed and freely-moving particles, and the heat conduction through the particles further influences the convection in a moving-particle system as the heat conductivity ratio is increased.

These results highlight the effect of temperature distributions within the particles as well as liquid on the overall heat transfer performance in the multiphase flow. 


\section{References}

1 Ishii, M. and Hibiki, T., Thermo-fluid Dynamics of Two-Phase Flow Eyrolles (1975)

2 McKenna, T.F., Spitz, R. and Cokljat, D., "Heat Transfer from Catalysts with Computational Fluid Dynamics", Reactors, Kinetics, and Catalysis 45-11, pp.2392-2410 (1999)

3 Gan, H., Feng, J.J. and Hu, H.H., "Simulation of the sedimentation of melting solid particles", International Journal of Multiphase Flows 29, pp.751-769 (2003)

4 Gan, H., Chang, J., Feng, J. and Hu, H.H., "Direct numerical simulation of the sedimentation of solid particles with thermal convection", Journal of Fluid Mechanics 481, pp.385-411 (2003)

5 Yu, Z., Shao, X. and Wachs, A., "A fictitious domain method for particulate flows with heat transfer", Journal of Computational Physics 217, pp.424-452 (2006)

6 Iaccarino, G. and Moreau, S., "Natural and Forced Conjugate Heat Transfer in Complex Geometries on Cartesian Adapted Grids" Journal of Fluids Engineering 128, pp.838-846 (2006)

7 Kim, J., Kim, D. and Choi, H., "An immersed-boundary finite-volume method for simulation of flow in complex geometries", Journal of Computational Physics 171, pp.132-150 (2001)

8 Kim, J. and Choi, H., "An immersed-boundary finite-volume method for simulation of heat transfer in complex geometries", KSME International Journal 18, pp.1026-1035 (2004)

9 Pacheco, J.R., Pacheco-Vega, A., Rodić, T. and Peck, R.E., "Numerical Simulations of Heat Transfer and Fluid Flow Problems Using an Immersed-Boundary Finite-Volume Method on Nonstaggered Grids", Numerical Heat transfer, Part B 48, pp.1-24 (2005)

10 Pacheco-Vega, A., Pacheco, J. R. and Rodić, T., "A general scheme for the boundary conditions in convective and diffusive heat transfer with immersed boundary methods", Journal of Heat Transfer 129-11, pp.1506-1516 (2007)

11 Ren, W.W., Shu, C., Wu, J. and Yang, W.M., "Boundary condition-enforced immersed boundary method for thermal flow problems with Dirichlet temperature condition and its applications", Computers \& Fluids 57, pp.40$51(2012)$

12 Zhang, N., Zheng, Z.C. and Eckels, S., "Study of heat-transfer on the surface of a circular cylinder in flows using an immersed-boundary method", International Journal of Heat and Fluid Flow 29, pp.1558-1566 (2008)

13 Ueyama, A., Moriya, S., Nakamura, M. and Kajishima, T., "Immersed Boundary Method for Liquid-Solid TwoPhase Flow with Heat Transfer (in Japanese)", Transaction of JSME Series B 77-775, pp.803-814 (2011)

14 Kajishima, T., Takiguchi, S., Hamasaki, H. and Miyake, Y., "Turbulence structure of particle-laden flow in a vertical plane channel due to vortex shedding", JSME International Journal Series B 44-4, pp.526-535. (2001)

15 Yuki, Y., Takeuchi, S. and Kajishima, T., "Efficient immersed boundary method for strong interaction problem of arbitrary shape object with the self-induced flow", Journal of Fluid Science and Technology 2-1, pp.1-11 (2007)

16 Kajishima, T. and Takiguchi, S., "Interaction between particle clusters and fluid turbulence", International Journal of Heat and Fluid Flow 23-5, pp.639-646 (2002)

17 Kajishima, T., "Influence of particle rotation on the interaction between particle clusters and particle-induced turbulence", International Journal of Heat and Fluid Flow 25-5, pp.721-728 (2004)

18 Nishiura, D., Shimosaka, A., Shirakawa, Y. and Hidaka, J., "Hybrid Simulation of Hindered Settling Behavior of Particles Using Discrete Element Method and Direct Numerical Simulation (in Japanese)", Kagaku Kogaku Ronbunshu 32-4, pp.331-340 (2006) 
19 Kuwagi, K., Mikami, T. and Horio, M., "Numerical simulation of metallic solid bridging particles in a fluidized bed at high temperature", Power Technology 109, pp.27-40 (2000)

20 Tsuji,Y., Kawaguchi,T. and Tanaka,T., "Discrete particle simulation of two-dimensional fluidized bed", Powder Technology 77, pp.79-87 (1993)

21 Moukalled, F., and Darwish, M., "New Bounded Skew Central Difference Scheme, Part II: Application to Natural Convection in an Eccentric Annulus", Numerical Heat Transfer, Part B 31, pp.111-133 (1997)

22 Sadat, H., and Couturier, S., "Performance and Accuracy of a Meshless Method for Laminar Natural Convection", Numerical Heat Transfer, Part B 37, pp.455-467 (2000)

23 Pan, D., "An immersed boundary method on unstructured Cartesian meshes for incompressible flows with heat transfer", Numerical Heat Transfer, Part B 49, pp.277-297 (2006)

24 Takeuchi, S., Tsutsumi, T. and Kajishima, T., "Behaviours of dispersed particles and heat transfer in buoyantdriven two-phase flows with different solid-liquid heat conductivities", Proc. of 4th International Conference on Computational Methods (Gold Coast, Australia), Paper ID: 151, (2012)

25 Iwata, R., Kajishima, T. and Takeuchi, S., "Analysis of a rising bubble passing through array of particles by a coupled immersed-boundary and volume-of-fluid method (in Japanese)", Japanese Journal of Multiphase Flow, 23-5, pp.531-538 (2010)

\section{Acknowledgements}

The authors gratefully acknowledge the financial supports of Grant-in-Aid for Scientific Research (B) No. 23360085 and Grant-in-Aid for Young Scientist (A) No. 23686030 by the Japan Society for the Promotion of Science (JSPS). 